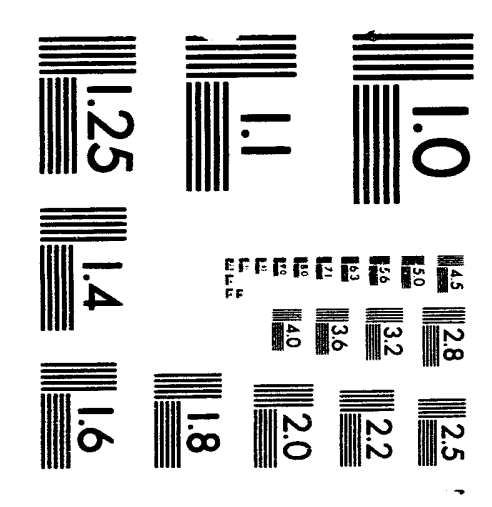



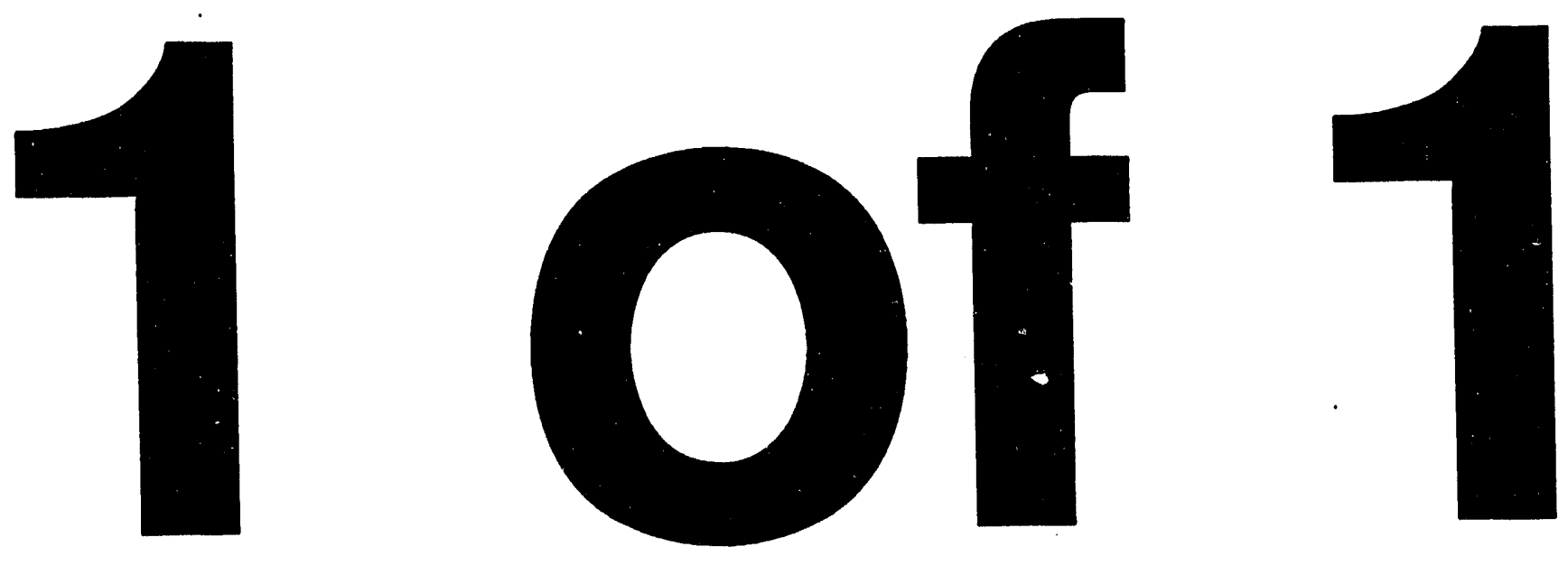


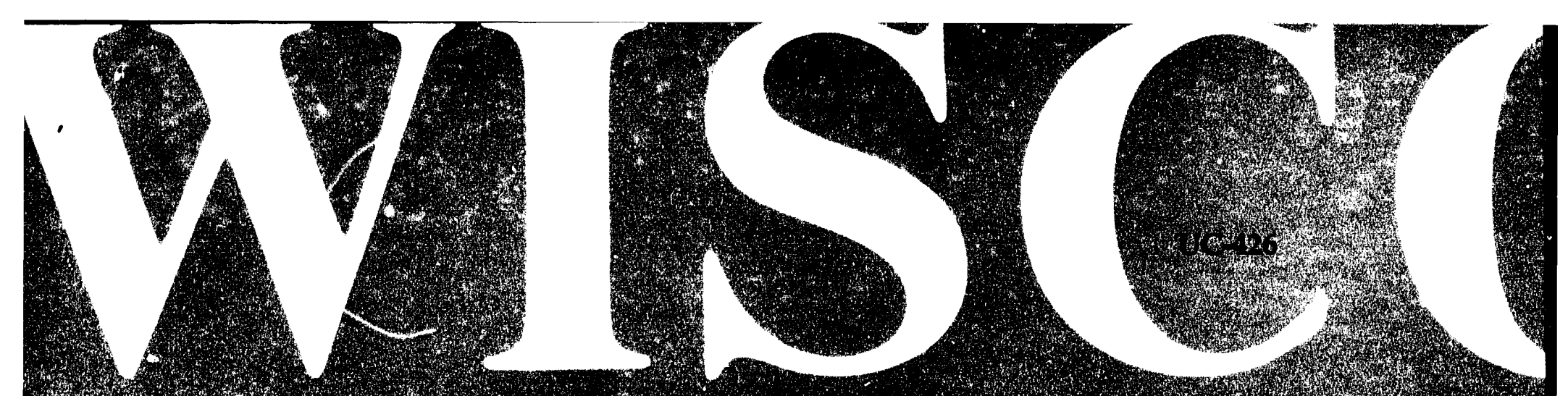

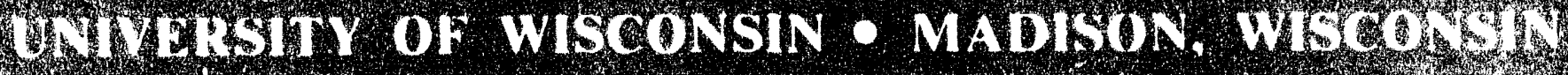

\section{PLASMA PHYSICS}

-

Fluctuation and Transport Reduction in a Reversed Field Pinch by Inductive Poloidal Current Drive

J.S. Sarff, S.A. Hokin, H. Ji, S.C. Prager, C.R. Sovinec

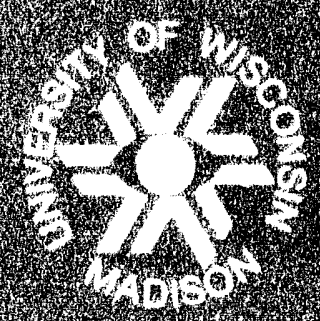

(x)

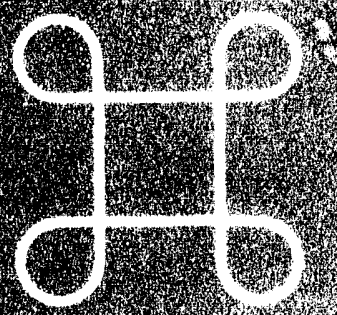

2.
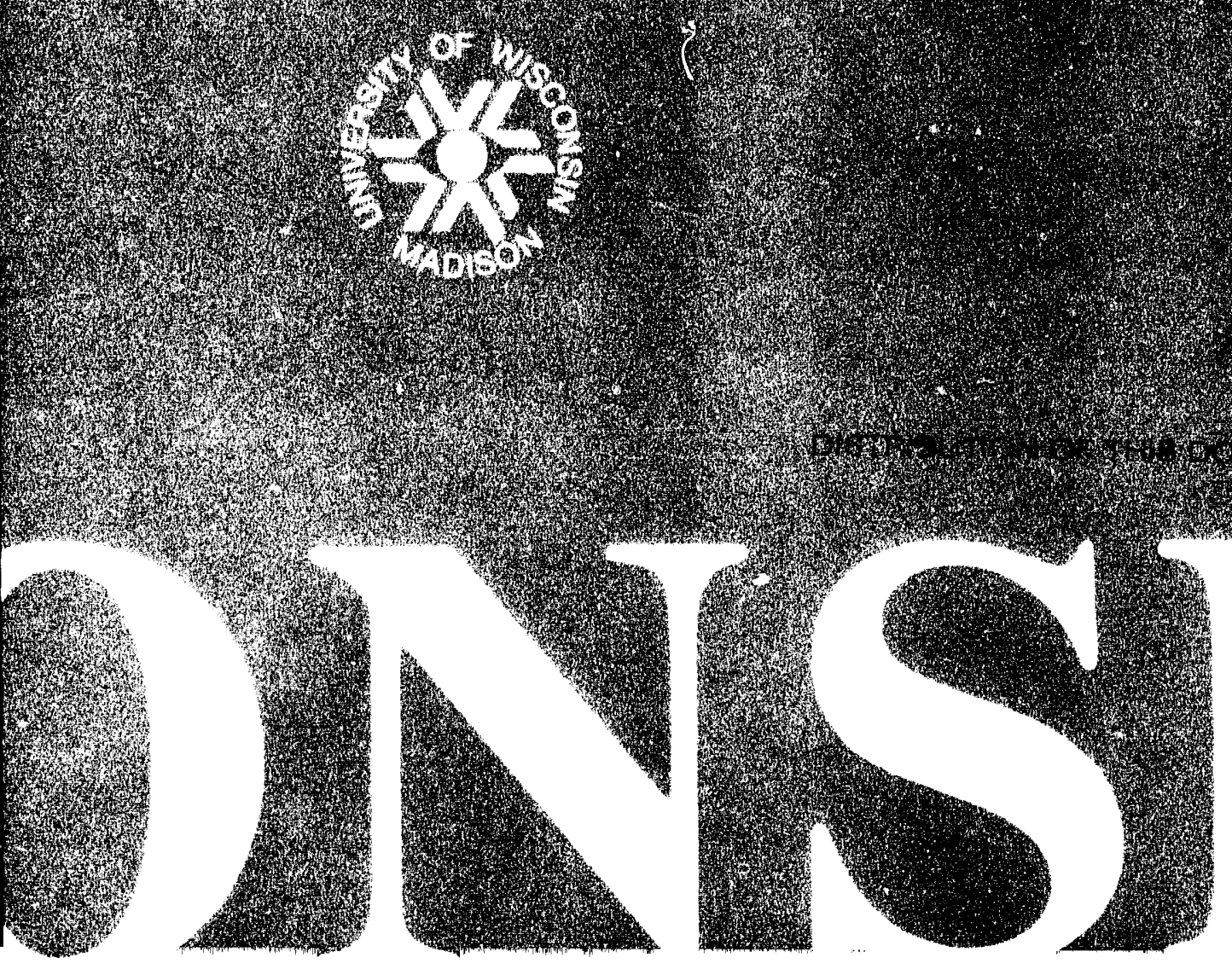

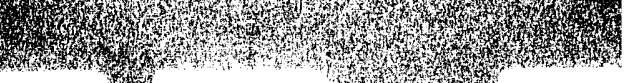




\section{NOTICE}

This report was prepared as an account of work sponsored by an agency of the United States Government. Neither the United States nor any agency thereof, nor any of their employees, makes any warranty, expressed or implied, or assumes any legal liability or responsibility for any third party's use or the results of such use of any information, apparatus, product or process ciisclosed in this report, or represents that its use by such third party would not infringe privately owned rights.

Printed in the United States of America

Available from

National Technical Information Service

U.S. Department of Commerce

5285 Port Royal Road

Springfield, VA 22161

NTIS Price codes

Printed copy: $\quad$ A02

Microfiche copy: A01 


\title{
Fluctuation and Transport Reduction in a Reversed Field Pinch by Inductive Poloidal Current Drive
}

\author{
J. S. Sarff, S. A. Hokin, H. Ji, S. C. Prager, and C. R. Sovinec \\ University of Wisconsin, Madison, WI 53706
}

An auxiliary poloidal inductive electric field applied to a reversed field pinch plasma reduces the current density gradient, slows the growth of $m=1$ tearing fluctuations, suppresses their associated sawteeth, and doubles the energy confinement time. Small sawteeth occur in the improved state but with $m=0$ precursors. By requiring a change of toroidal flux embedding the plasma, inductive poloidal current profile drive is transient, but the improvement encourages the program of RFP transport suppression using current profile control. 
The search for solutions to the problem of fluctuation-induced transport in magnetically confined plasmas absorbs the efforts of many plasma researchers. Proposed strategies based on notions of plasma behavior are often tested, but improved confinement usually results unexpectedly. In the case of the reversedfield pinch (RFP), magnetic-fluctuation-induced transport dominates other loss mechanisms. The fluctuation amplitude is typically one percent of the mean field, ${ }^{1}$ and the estimated convective energy loss by stochastic magnetic field diffusion easily accounts for the observed global energy flux. ${ }^{2}$ Recent measurements of the magnetic-fluctuation-induced electron energy flux in the Madison Symmetric Torus (MST) directly identify large transport associated with the magnetic fluctuation. ${ }^{3}$

More than $90 \%$ of the RFP magnetic fluctuation results from several $m=1$, $n \sim 2 R / a$ tearing (or resistive kink) instabilities. An understanding of these MHD fluctuations, ${ }^{4}$ which correlate with the energy transport, led researchers to propose methods for their elimination. Tearing fluctuation stems from the current density gradient, so the proposals employ electrostatic ${ }^{5}$ or $\mathrm{rf}^{6,7}$ poloidal current drive in the outer region of the plasma, eliminating the need for fluctuation-dynamo sustainment of the RFP.

In this Letter we describe the first observation of reduced transport resulting from current profile control in an RFP. We call this experiment pulsed poloidal current drive (PPCD) as it uses inductive methodology. Unlike electrostatic or rf current drive, PPCD is transient since it requires a change of toroidal flux embedding the plasma. PPCD exemplifies a rare and successful progression from the identification of destructive turbulence to a theoretical understanding which motivates a corrective measure.

The PPCD experiment is performed in $\mathrm{MST}^{8}$ a large reversed field pinch with major radius $R=1.5 \mathrm{~m}$, minor radius $a=0.52 \mathrm{~m}$, toroidal plasma current 
$I_{\phi} \leq 700 \mathrm{kA}$, and poloidal beta $\beta_{\theta}-10 \%$. Fig. 1 summarizes a PPCD experiment in which a small high voltage capacitor bank drives a fast current pulse in the toroidal field winding to induce a poloidal electric field $E_{\theta}$. The pulse is initiated at $t=12 \mathrm{~ms}$, marked by the vertical dashed lines. The one-turn poloidal $V_{\theta}$ and toroidal $V_{\phi}$ surface voltages shown in Fig. 1(a) generate the poloidal $I_{\theta}$ and toroidal $I_{\phi}$ plasma currents shown in Fig. $1(\mathrm{~b})$, although strong coupling prevents identifying $V_{\theta}$ only with $I_{\theta}$ and vice versa. To direct $E_{\theta}$ for current profile flattening, the volume average toroidal field $\left\langle B_{\phi}\right\rangle$ and the toroidal field at the wall $B_{\phi w}$ must decrease as in Fig. 1(c). PPCD increases $I_{\theta}=2 \pi R\left(B_{0}-B_{\phi w}\right) / \mu_{0}$, the poloidal plasma current between the magnetic axis and plasma edge which is inferred from $\oint \mathbf{B} \cdot \mathbf{d l}$ on the magnetic axis. (The axis magnetic field $B_{0}$ is estimated using an RFP equilibrium model. ${ }^{9}$ ) Note that $V_{\theta}$ is nonzero as $I_{\phi}$ increases since toroidal flux is generated by dynamo action. The Fig. 1 data are averages for eleven PPCD plasmas.

Since tearing instability results from the gradient in $J_{\|} / B$, we fit the experiment to the three parameter equilibrium model ${ }^{10}$

$$
\nabla \times \mathbf{B}=\lambda_{v}\left(1-r^{x}\right) \mathbf{B}+\left(\beta_{v} / 2 B^{2}\right) \mathbf{B} \times \nabla p
$$

to estimate the shape of the (normalized) parallel current profile $\lambda(r)=\mu_{0} a \mathrm{~J} \cdot \mathbf{B} / B^{2}$. Large $\alpha$ corresponds to a flat parallel current density profile. $\beta_{0}=2 \mu_{0} p_{0} / B_{0}^{2}$ is the central beta value, and the pressure profile is assumed quadratic $p(r)=1-r^{2}$. In general, the perpendicular current details weakly affect the parallel current fit.

PPCD flattens the current profile in degree comparable to a sawtooth oscillation "crash".11 This comparison benchmarks PPCD since in a sawtooth crash the plasma self-flattens its unstable current profile. To illustrate, we plot the $F-\Theta$ trajectory where $F$ is the cylindrical reversal parameter, $F \equiv B_{\phi w} /\left\langle B_{\phi}\right\rangle$, and $\Theta$ is the cylindrical pinch parameter, $\Theta \equiv B_{\theta w} /\left\langle B_{\phi}\right\rangle . B_{\theta w}$ and $B_{\phi w}$ are the poloidal 
and toroidal magnetic field at the plasma surface respectively. ( $F$ and $\Theta$ are alternate parameters to $\lambda_{0}$ and $\alpha$.) The PPCD $F-\Theta$ trajectory is shown in Fig. 2 for $12.2 \leq t \leq 19.5 \mathrm{~ms}$ data in Fig. 1. Solutions to Eq. 1 for $\beta_{\theta}=2 \mu_{0}\langle p\rangle / B_{\theta} w^{2}=10 \%$ are indicated by the dashed contours of constant $\alpha$. For comparison, the average $F-\Theta$ trajectory of the sawtooth oscillation which crashed at $t \approx 10 \mathrm{~ms}$ in the eleven PPCD discharges is also shown in Fig. 2. The PPCD phase gradually terminates as the current profile again peaks and becomes sawtooth unstable when $\alpha \lesssim 2$. A series of unusually large sawteeth starting around $t \approx 18 \mathrm{~ms}$ cause the plasma to relax toward normal RFP equilibria.

By flattening the current profile, PPCD slows the growth of $m=1$ fluctuations and suppresses their associated sawteeth. Magnetic fluctuations are measured in MST with arrays of magnetic pickup sensors $\left(B_{\theta}, B_{\phi}, B_{r}\right)$ attached to the inner vacuum vessel surface. In this work, toroidal mode $n \leq 15$ spectra are derived from 32 equally-spaced magnetic pickup sensors. The most active modes in a typical $n$ spectrum of $\widetilde{B}_{\theta}$ from a single PPCD plasma are shown in Fig. 3. Before the application of the pulse, the spectrum exhibits the usual precursory growth of the $n=5-10, m=1$ modes associated with the sawtooth cycle. When PPCD is applied, their growth is dramatically slowed. The spatial root-meansquare (RMS) fluctuation amplitude $\tilde{B}_{\theta r m s}=\sqrt{\Sigma b_{\theta n}^{2}}$ for $n \leq 15$ is shown in Fig. 4(a). The maximum amplitude is about three times the minimum amplitude between sawtooth crash events; PPCD maintains this minimum value for several milliseconds. The lack of sawtoothing decreases the average fluctuation amplitude by $25 \%$ during PPCD, indicated by the (time-smoothed) plot of the shot-averaged RMS fluctuation overlaying the single shot record in Fig. 4(a).

Although PPCD eliminates sawteeth preceded by $m=1$ fluctuation, different sawteeth occur during PPCD. Like conventional sawteeth, they correlate with decreases in the soft X-ray flux (Fig. 4(b)) and increases in toroidal 
flux, but the amplitude changes are small. The $n$ spectra reveal increased precursory activity in the $n=1-3$ modes, not in the band $n=5-10$. This activity, if resonant, corresponds to $m=0$ fluctuation since the safety factor satisfies $|q(r)| \leqslant 1 / 5$, even during PPCD. Simultaneous spectral measurements of both $\widetilde{B}_{\phi}$ and $\widetilde{B}_{\theta}$ show $\widetilde{B}_{\phi} / \widetilde{B}_{\theta} \gtrsim 5$, consistent only with $m=0$ instability since the sensors are located in a current-free region. ${ }^{12}$ In addition to sawtoothing, the low $n$ mode amplitudes steadily grow to pre-crash values when PPCD is increased to the level where $\left\langle B_{\phi}\right\rangle$ is halved by the pulse. In moderate amplitude PPCD, the steady $m=0$ growth is avoided. Interestingly, MHD modeling of PPCD using the DEBS code ${ }^{13}$ predicts reduced, not stabilized, $m=1$ fluctuation as in the experiment, but it fails to predict enhanced $m=0$ fluctuation.

PPCD doubles the energy confinement by halving the Ohmic input power while slightly increasing the stored thermal energy. The solid line curves in Fig. 5 show shot-averaged waveforms of the central chord electron density $n_{e}$, the charge-exchange ion temperature $T_{i}, P_{\text {Ohmic }}\left(E_{\theta}\right.$ included), and the total radiated power $P_{\text {rad }}$ (bolometrically measured) for the eleven PPCD plasmas. Also shown are soft $X$-ray, $H_{\alpha}$, and near-infrared Bremsstrahlung $\left(\propto n^{2} Z_{e f f} / \sqrt{T_{e}}\right)$ radiation measurements. The dashed line waveforms are for a set of eight discharges with PPCD turned off. These were operated identically to PPCD except a small gas puff was injected at $t=10 \mathrm{~ms}$ to mimic a modest density increase during PPCD. The electron temperature $T_{\mathfrak{e}}$ is estimated from a self-consistent analysis of the density $n_{e}, \mathrm{Si}(\mathrm{Li})$ detector $\mathrm{X}$-ray energy spectra, and two different thickness beryllium foil filtered X-ray measurements. At $t=17 \mathrm{~ms}, T_{e} \approx 250 \mathrm{eV}$ with PPCD and $T_{e} \approx 200 \mathrm{eV}$ without PPCD; the latter is consistent with the most recent database of MST Thomson scattering data. ${ }^{14}$ (The MST Thomson scattering diagnostic was inoperable during this PPCD experiment.) 
Assuming flat temperature and parabolic density profiles (consistent with the four interferometer chords inside $r / a=0.6$ ), the energy confinement time at $t=17 \mathrm{~ms}$ is $\tau_{E}=3 \pi^{2} a^{2} R\left\langle n_{e}\right\rangle\left(T_{e}+T_{i}\right) / P_{\text {Ohmic }} \approx 1.0 \mathrm{~ms}$ without PPCD and $\tau_{E} \approx 2.2 \mathrm{~ms}$ with PPCD. In standard RFP operation, $\tau_{E}$ scaling 14 in MST is weakly dependent on $I_{\phi}$ and $n_{e}$, varying little about $\tau_{E}=1 \mathrm{~ms}$. Because the stored magnetic energy changes during PPCD, the calculation of $P_{\text {Ohmic }}$ was cross-checked using several equilibrium models.9,10,15 The Polynomial Function Model ${ }^{9}$ calculation, shown in Fig. 5(c), gives slightly larger values for $P_{\text {Ohmic }}$ than the other models.

The particle confinement time $\tau_{p}$ also increases during PPCD. This is indicated by the $40 \%$ decrease in $H_{\alpha}$ emission and moderate rise in $n_{e}$. Particle transport modeling estimates $\tau_{p}$ increased by a factor of about 1.7 during PPCD.

PPCD reduces the anomalous plasma resistance. The change in toroidal plasma resistance from increased poloidal field line twist during PPCD almost balances the change from reduced classical resistivity $\eta \propto \mathrm{Z}_{e f f} / T_{e}^{3 / 2}$. ( $T_{e}$ increases, and $Z_{\text {eff }}$ decreases by about $20 \%$.) Therefore most of the reduction in $P_{\text {Ohmic }}$ results from a $40 \%$ decrease in anomalous plasma resistance ${ }^{16}$ during PPCD. This conclusion is sensitive to the error in the $T_{e}$ measurement, but a $50 \% T_{e}$ increase or a dramatic $T_{e}$ profile change is required to explain the $P_{\text {Ohmic }}$ reduction classically. From the combined reductions in $Z_{\text {eff }}$ and anomalous effects, the multiplier of the $Z=1$, flat temperature profile resistance decreases from 3 to 1.5 . Typical of RFP plasmas, the ion temperature exceeds expectations for collisional heating by electrons. If the anomalous input power heats the ions, as often assumed, then the reduced anomalous resistance and unchanged ion temperature during PPCD imply much reduced ion thermal loss.

Improved confinement during PPCD depends on the condition of the vacuum vessel wall. Clear improvement occurs with a boronized wall. (Solid target boronization is used in MST. ${ }^{17}$ ) Without boronization, enhanced impurity 
influx coincides with the PPCD pulse. Even with boronization, if the pulse is applied well after current peak, impurity-injection-free PPCD is difficult to obtain.

In summary, inductive poloidal current drive flattens the current density profile, slows the growth of $m=1$ tearing fluctuations, suppresses their associated sawteeth, and doubles energy confinement. The particle confinement time also improves. A reduction in anomalous plasma resistance suggests PPCD reduces the dynamo effect. The improved plasma state exhibits small sawteeth, but they are preceded by $m=0, n \sim 1$ instability rather than $m=1, n \sim 6$ instability.

These results strongly encourage the program of fluctuation and transport suppression using current profile control techniques in the RFP. Although the PPCD experiment falls short of the ultimate goal of eliminating tearing fluctuation, clear correlation exists between improved confinement, current profile flattening, and modest fluctuation suppression. If the conductive energy loss scales as $\chi \sim B_{r}^{2}$, then the $25 \%$ reduction in the average fluctuation amplitude predicts a $45 \%$ reduction in the conducted power. The measured improvement in confinement is consistent with this number, but the conducted power may even be smaller since the PPCD improved state is relatively more radiative. The plasma core could be on the verge of experiencing closed flux surfaces.

The authors are grateful for the assistance of the MST group, especially A. Almagri, M. Cekic, J. Frank, D. Den Hartog, J. Laufenberg, K. Mirus, D. Sinitsyn, W. Shen, and C. Sprott. We also acknowledge P. Figgliozi for computational assistance. This work was supported by the US DoE. 


\section{REFERENCES}

1For example, V. Antoni and S. Ortolani, Plasma Phys. 25, 799 (1983);

D. Brotherton-Ratcliffe, C. G. Gimblett, and I. H. Hutchinson, Plasma Physics and Controlled Fusion 24, 59 (1984); R. J. La Haye et al., Phys. Fluids 27, 2576

(1984); A. Almagri et al., Phys. Fluids B 4, 4080 (1992); P. R. Brunsell et al., Phys. Fluids B 5, 885 (1993).

${ }^{2}$ For example, I. H. Hutchinson et al., Nucl. Fusion 24, 59 (1984) and K. Hattori et al., Phys. Fluids B 3, 3111 (1991).

${ }^{3}$ G. Fiksel, S. C. Prager, W. Shen, and M. Stoneking, submitted to Phys. Rev. Lett.

4Y. L. Ho and G. Craddock, Phys. Fluids B 3, 721 (1991) and references therein.

5Y. L. Ho, Nucl. Fusion 31, 341 (1991).

6S. Shiina et al., in Proceedings of the International School of Plasma Physics Workshop on the Physics of Alternative Magnetic Confinement Schemes, Varenna, Italy

(Societá Italiana di Fisica, Varenna, 1990), p. 903.

${ }^{7}$ E. Uchimoto et al., Bull. Amer. Phys. Soc. 38, 1979 (1993).

${ }^{8}$ R. N. Dexter, D. W. Kerst, T. H. Lovell, S. C. Prager, and J. C. Sprott, Fusion Technol. 19, 131 (1991).

9J. C. Sprott, Phys. Fluids 31, 2266 (1988).

10V. Antoni et al., Nucl. Fusion 26, 1711 (1986).

11R. G. Watt and R. A. Nebel, Phys. Fluids 26, 1168 (1983); S. Hokin et al., Phys. Fluids B 3, 2241 (1991).

$12_{i} \mathbf{k} \times \mathbf{B}=0$ implies $n b_{\theta m n} / R=m b_{\phi m n} / a$ for each Fourier mode $\mathbf{b}_{m n}$. In a cylinder, $b_{\theta 0 n}$ vanishes, but toroidal effects allow finite $b_{\theta 0}$ for " $m=0$ " instability.

${ }^{13}$ D. D. Schnack et al., J. Comput. Phys. 70, 330 (1987).

14S. Hokin et al., in Contolled Fusion and Plasma Physics, Proceedings of the 20th European Conference, Lisbon, 1993 (European Physical Society), Vol. II, p. 475. 
${ }^{15}$ K. F. Schoenberg, R. F. Gribble, and J. A. Phillips, Nucl. Fusion 22, 1433 (1982). ${ }^{16}$ Traditionally "anomalous" refers to $E_{\|} \neq \eta J_{\|}$for classical $\eta$. This anomaly need not be mysterious given a dynamo electric field, e.g., $\langle\tilde{\mathbf{V}} \times \widetilde{\mathbf{B}}\rangle_{\|}$.

17D. J. Den Hartog et al., J. Nucl. Mat. 200, 177 (1993). 

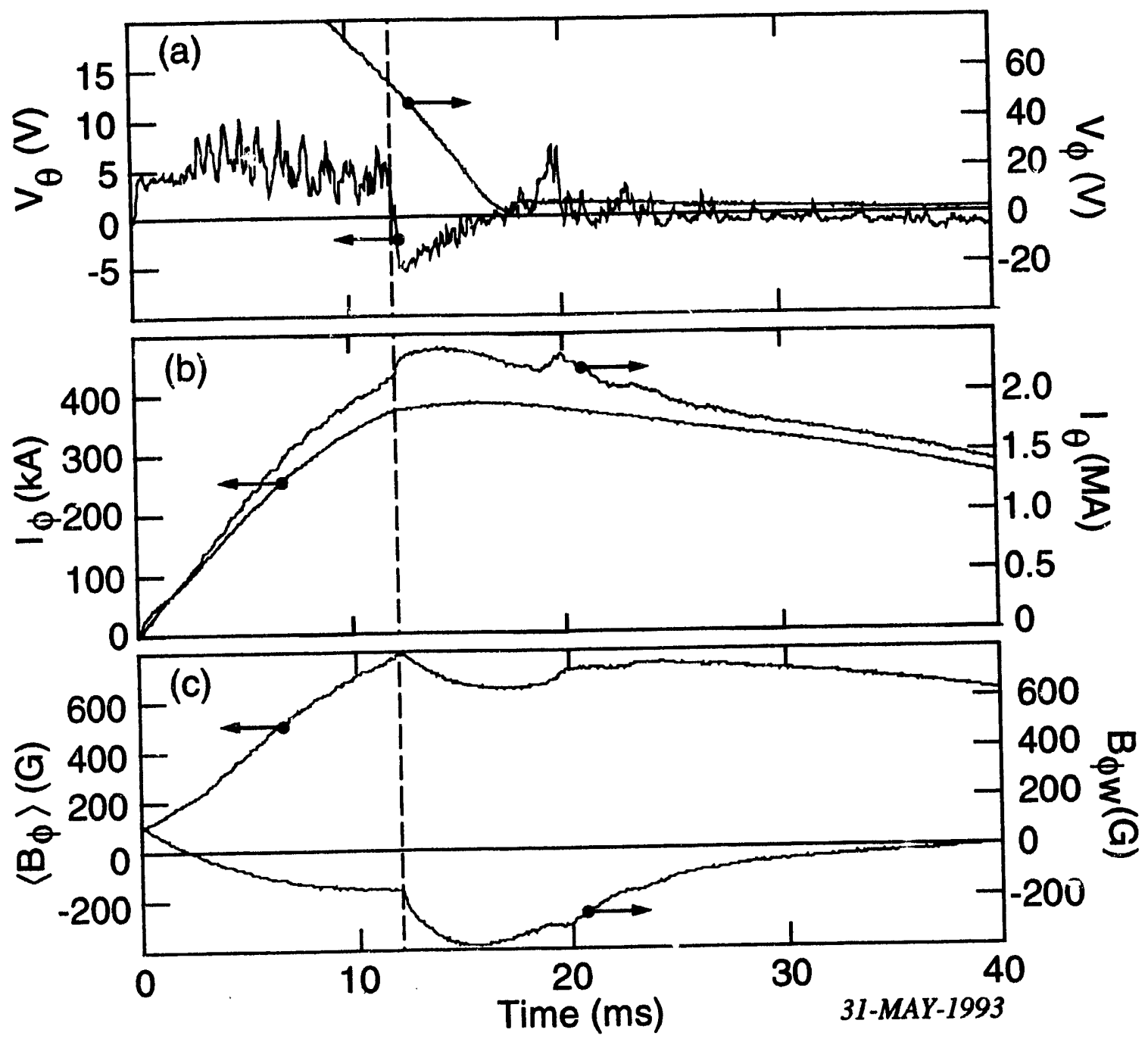

Fig. 1 Shot-averaged waveforms of (a) the surface voltages, (b) the plasma current, and (c) the average and wall toroidal field. 


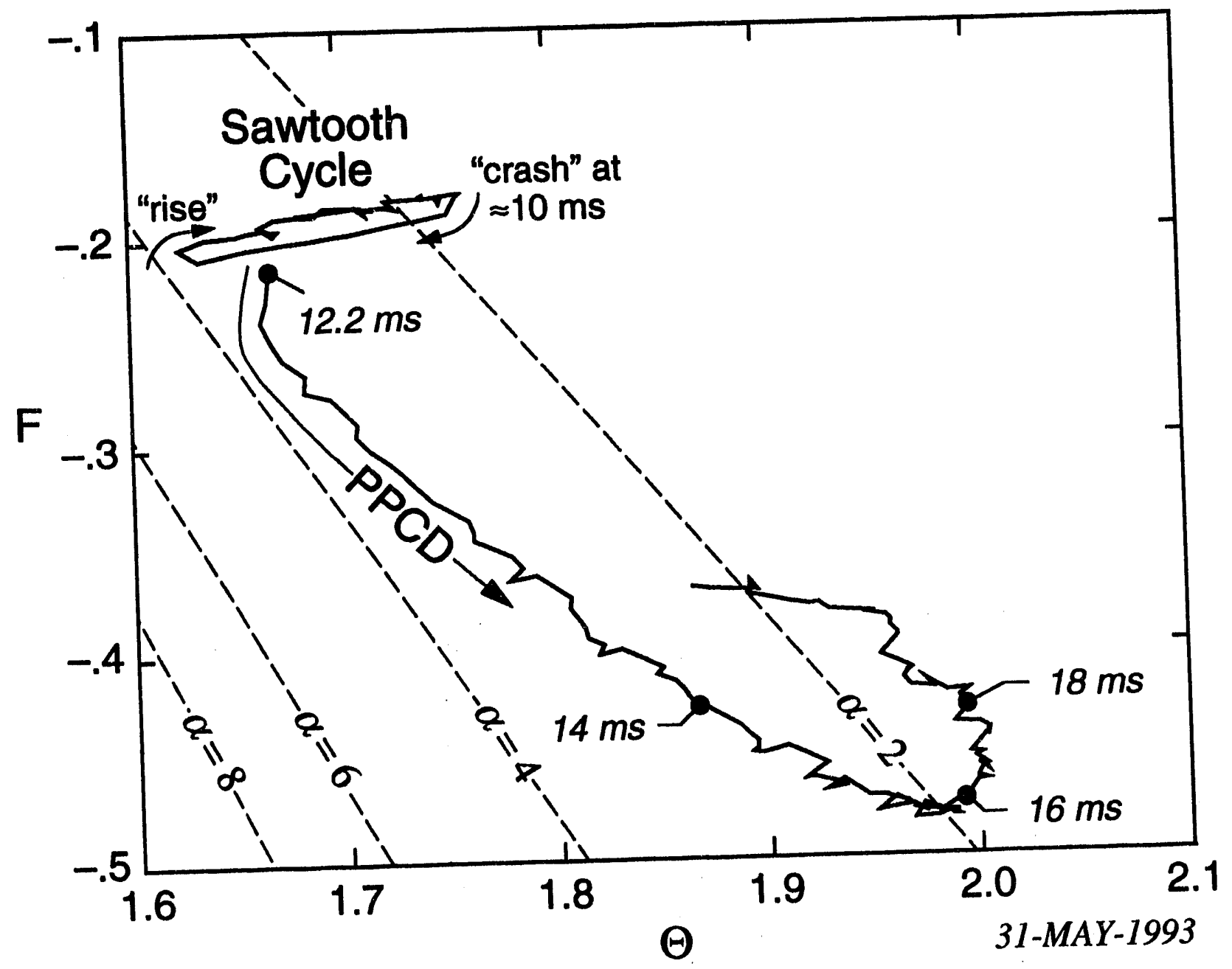

Fig. 2 Shot-averaged F- $\Theta$ trajectories during PPCD and a sawtooth oscillation. 


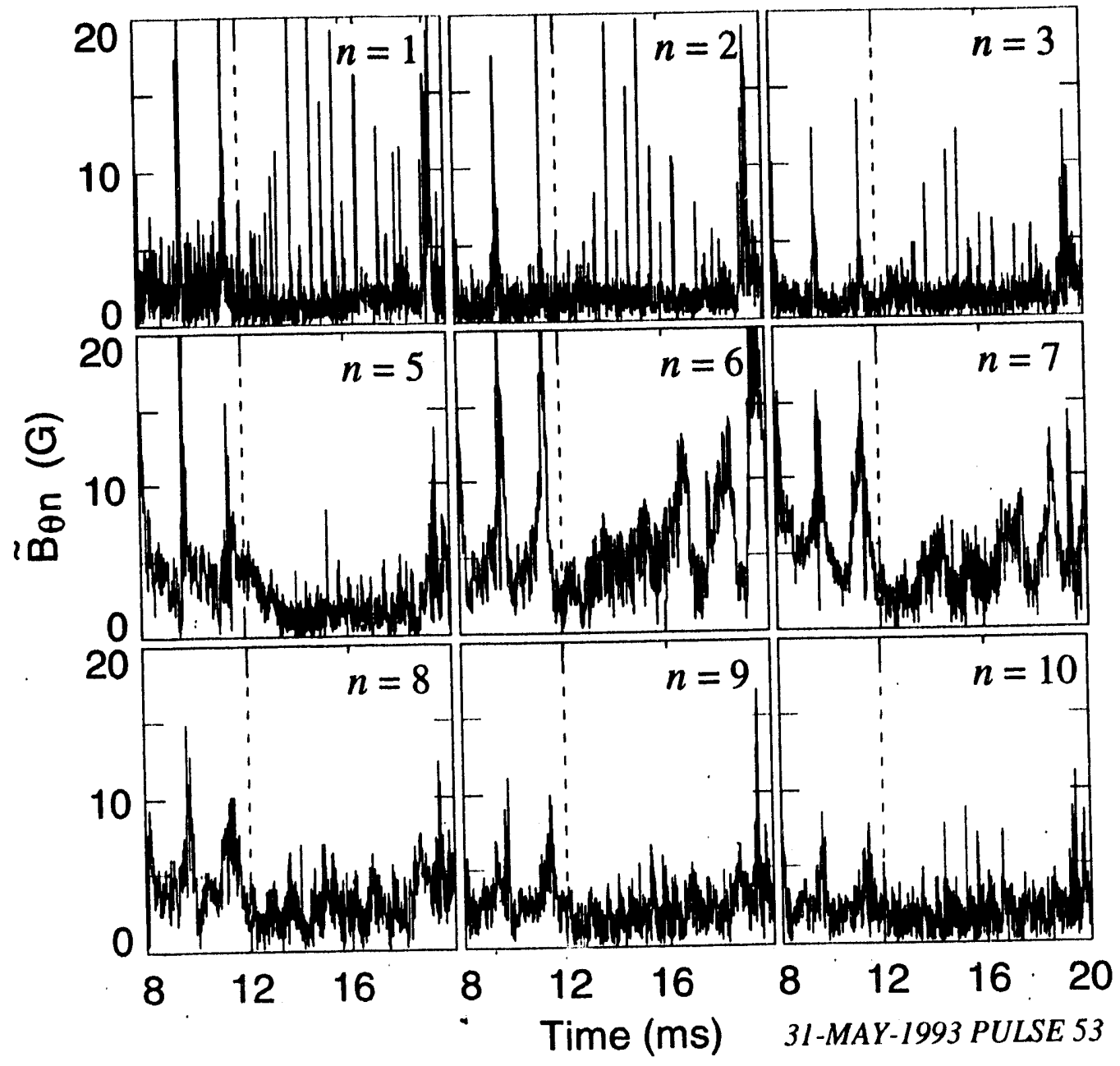

Fig. 3 Dominant modes of a single shot $\mathbb{B} \theta$ toroidal $n$ spectrum during PPCD. 


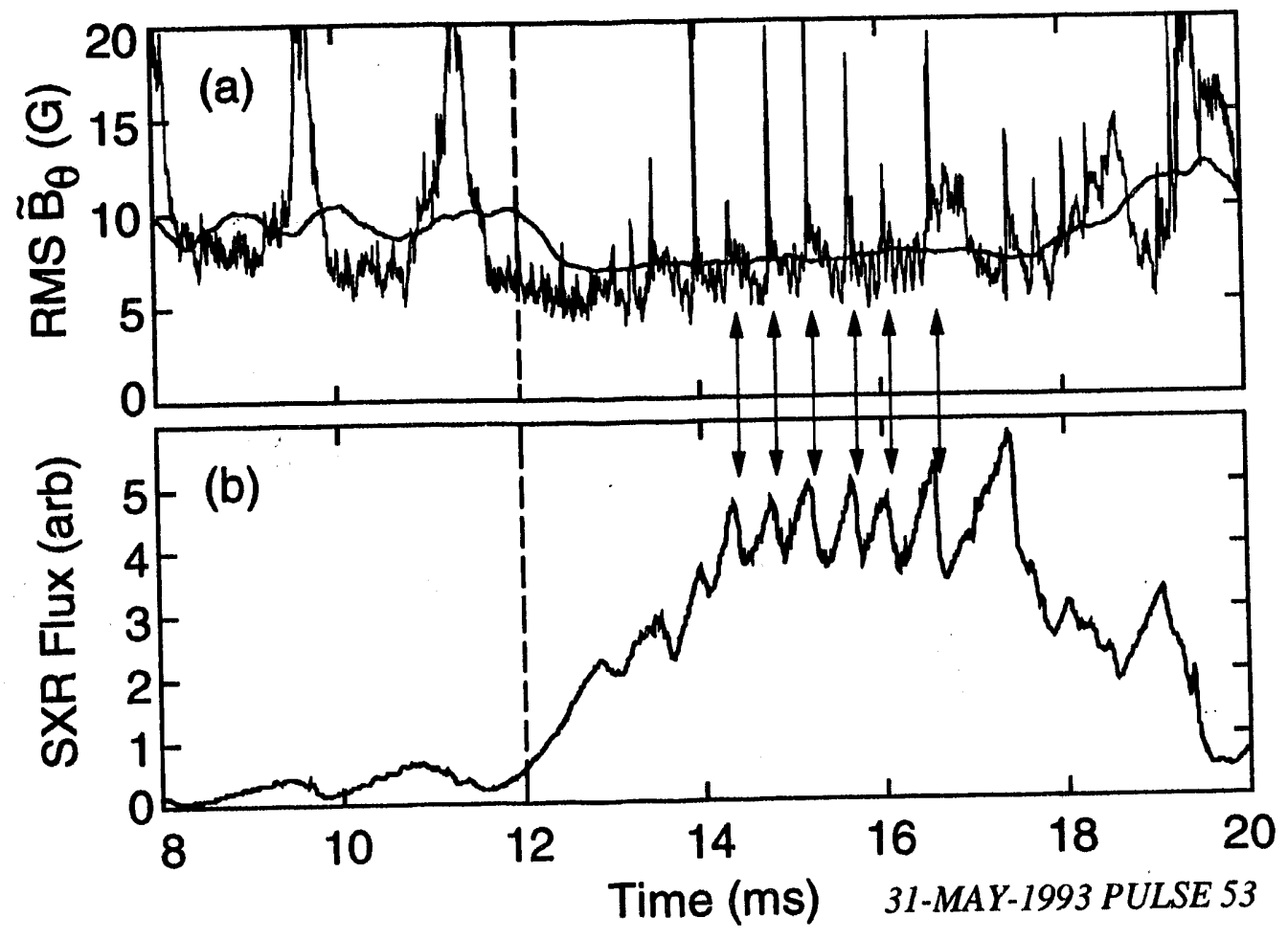

Fig. 4 (a) The spatial RMS fluctuation for the spectrum in Fig. 3. The smooth curve is the shot-averaged RMS fluctuation. (b) Central-chord soft X-ray emission. Arrows mark small sawteeth during PPCD. 

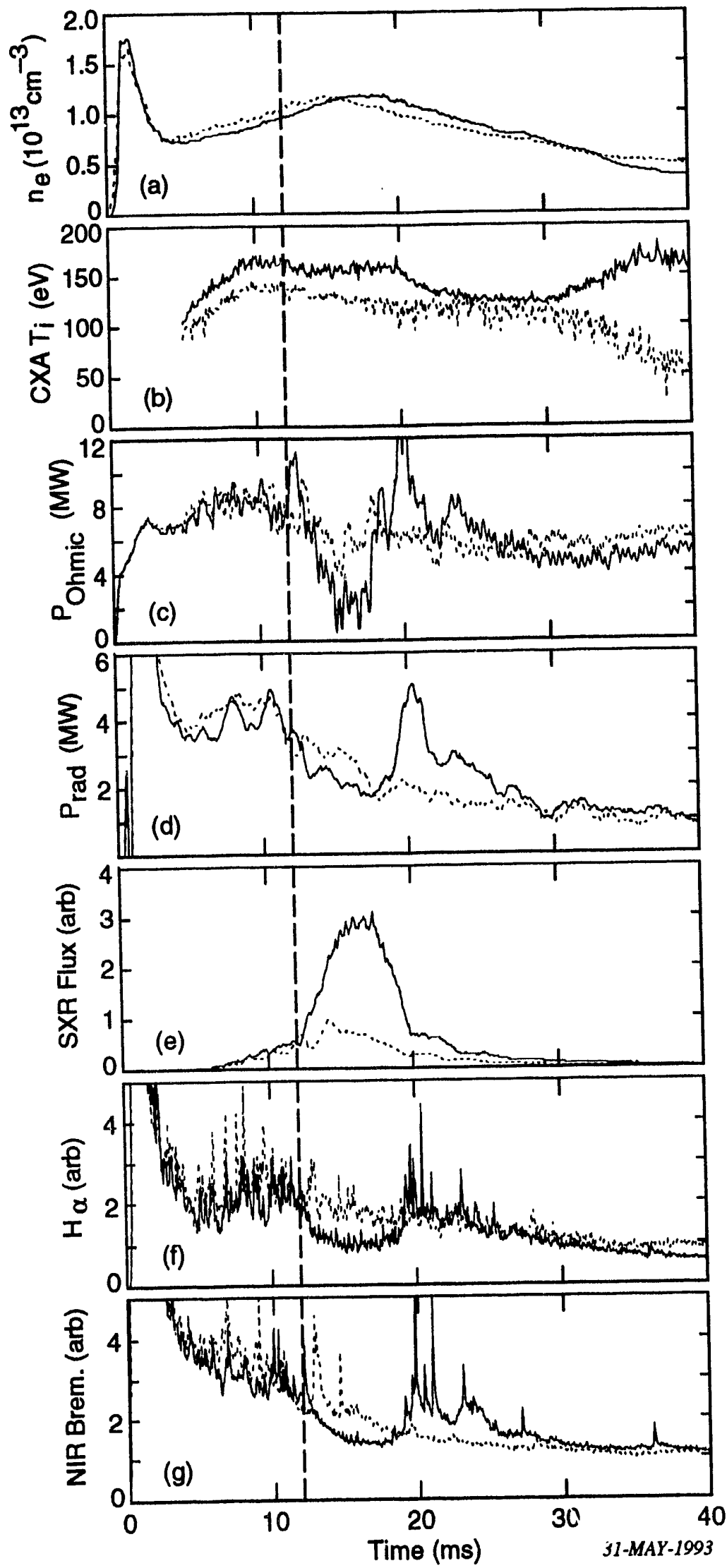

Fig. 5 Shot-averaged waveforms of (a) the central chord line-averaged electron density, (b) the ion temperature, (c) the Ohmic input power, (d) the total radiated power, (e) soft $\mathrm{X}$-rays, (f) $\mathrm{H} \alpha$ emission, and (g) Bremsstrahlung radiation. 


\section{EXTERNAL DISTRIBUTION IN ADDITION TO UC-20}

S.N. Rasband, Brigham Young University

T. Dolan, EG\&G Idaho, Inc.

R.A. Moyer, General Atomics

J.B. Taylor, Institute for Fusion Studies, The University of Texas at Austin

E. Uchimoto, University of Montana

F.W. Perkins, PPPL

O. Ishihara, Texas Technical University

M.A. Abdou, University of California, Los Angeles

R.W. Conn, University of California, Los Angeles

P.E. Vandenplas, Association Euratom-Etat Belge, Belgium

Centro Brasileiro de Pesquisas Firicas, Brazil

P. Sakanaka, Institute de Fisica-Unicamp, Brazil

Mme. Monique Bex, GANIL, France

J. Radet, CEN/CADARACHE, France

University of Ioannina, Greece

S. Ortolani, Istituto Gas Ionizzati, EURATON-ENEA-CNR Association, Italy

R. Andreani, Associazione EURATOM-ENEA sulla Fusione, Italy

Plasma section, Energy Fundamentals Division Electrotechnical Laboratory, Japan

Y. Kondoh, Gunma University, Kiryu, Gunma, Japan

H. Toyama, University of Tokyo, Japan

Z. Ypsjoda. University of Tokyo, Japan

FOM-Instituut voor Plasmafysica "Rijnhuizen," The Netherlands

Z. Ning, Academia Sinica, Peoples Republic of China

P. Yang, Shandong University, Peoples Republic of China

S. Zhu, University of Science \& Technology of China, People's Republic of China

I.N. Bogatu, Institute of Atomic Physics, Romania

M.J. Alport, University of Natal, Durban, South Africa

R. Storer, The Flinders University of South Australia, South Australia

B. Lehnert, Royal Institute of Technology, Sweden

Librarian, CRPP, Ecole Polytechnique Federale de Lausanne, Switzerland

B. Alper, Culham Laboratory, UK

A. Newton, UK

2 for Chicago Operations Office

5 for individuals in Washington Offices

INTERNAL DISTRIBUTION IN ADDITION TO UC-20

80 for local group and file 

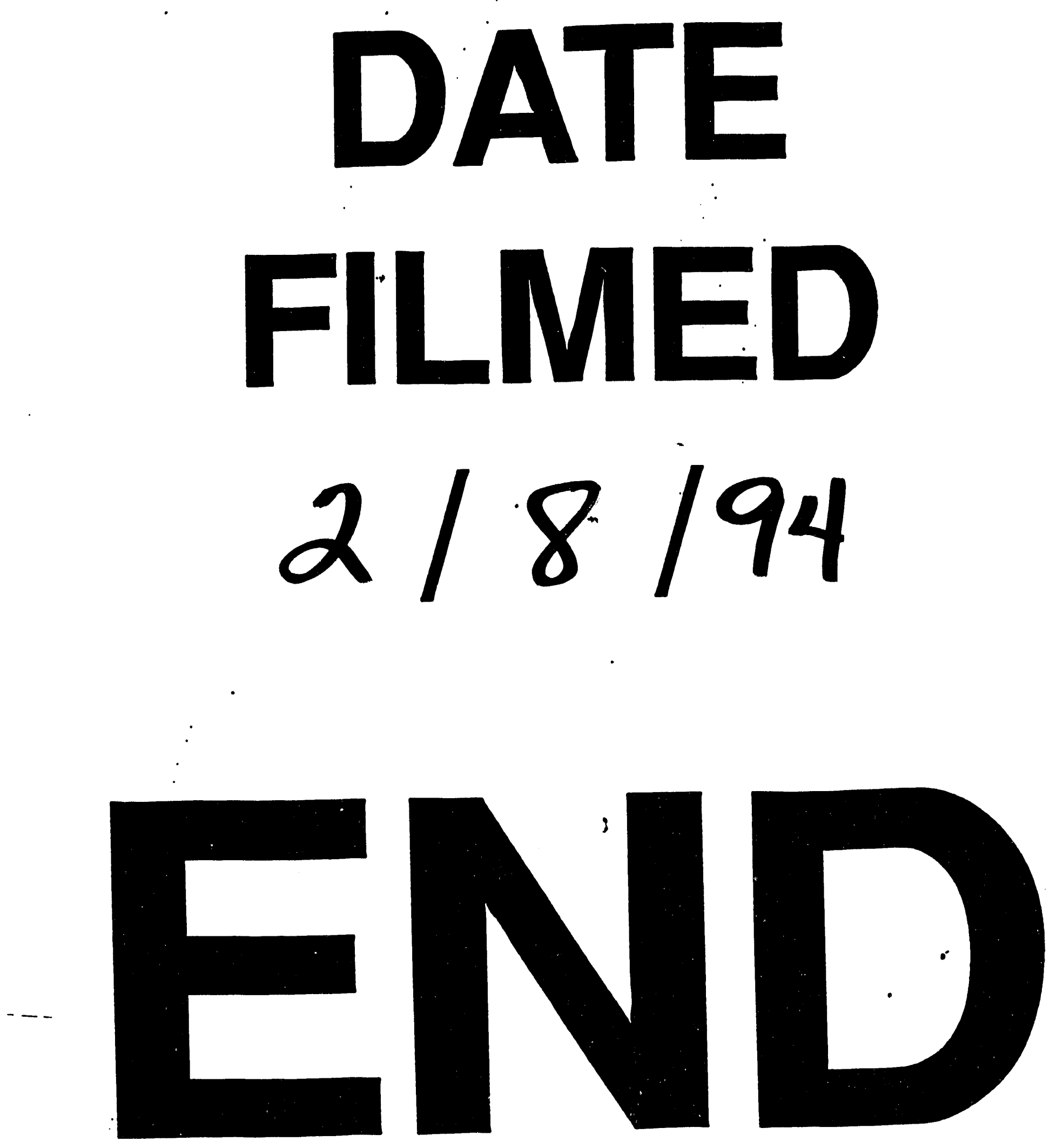
